\title{
Medical Diagnosis Expert System as Service in Cloud
}

\author{
A. B. Rajesh Kumar, G. V. Ramesh Babu, C. Phani Ramesh, P. Madhura, and M. Padmavathamma
}

\begin{abstract}
With the technological advancements in information technology, cloud computing provides several opportunities and services to users like storing personal information, accessing various web services for online transactions, online diagnosis systems etc.,. But security in cloud is biggest challenge which poses threat to individual privacy and misuse without knowledge of data owner. In such a situation, secure decision making based on patient information through web services using cryptographic techniques need to be adopted to protect sensitive data for diagnosis. In this paper, we propose a novel approach development of cloud model for medical diagnosis expert system in which patient access is divided into two ways. Patient needs to access openly and other is to be provided security in terms of encryption techniques which secure patient information through decision for diagnosis can be made based on personal information of the patient and to implement patient access to control using EAS [1] ID3 [2].
\end{abstract}

Index Terms-Cloud computing, SAAS, decision tree, ID3, expert system as service.

\section{INTRODUCTION}

Distributed computing environment that will collect authorized medical data store it securely within a networks to assist fast and efficient care. Cloud computing, health care services that benefits patients, doctors and hospitals for of medical data analysis for the purpose of decision based on Test reports [3]. In communication and information technology an increasing number of health services are available. Health care services provides patient's safety less health care cost and improving quality of diagnosis.[4] Despite number of changes by industry and a number of standards under development provides patient information. Security and privacy are major difficulties with respect to patient information. Patients are expected to treat as confidential. To address these issues cryptographic techniques can be implemented to maintains security for personal information. The advent of cloud computing has permitted us to host the software pack that analyses patient personal information as s/w as a services, [5] PAAS layer controls the execution of the software. In this paper, we propose a novel approach development of cloud model for medical diagnosis expert system in cloud which provides secure patient information through decisions based on lab test reports of the patient and to implement patient access control using expert system as service and ID3 [6], [7] .

Manuscript received February 2, 2013; revised April 10, 2013

The authors are with the Department of Computer Science, S.V. University, Tirupati, 517502, India (e-mail: a.b_rajeshkumar@yahoo.com, gvrameshbabu2002@yahoo.com, p_pramasa@yahoo.com,prof.padma@yahoo.com).

\section{RELATED WORK}

\section{A. Expert System}

Expert system is computer programs that are derived from computer science reveals called artificial intelligence (AI). In designed to diagnose patient data in developed to diagnose disease. Expert system includes both conventional such as DBMS and AI. [8] Medical Diagnosis DBMS are used to store and diagnosis patient personal information. In an interaction based expert system to assist the doctor for diagnosis process. Based on test reports and to bring out the appropriate information for consultation and with the results produce possible diagnosis and suggest treatment. To help doctors for disease associated with symptoms.

\section{B. Decision Trees}

Decision tree is a classification techniques that uses a series of questions or rules about the attributes of the class to classify data sets into classes. Training data set to establish a classifier is used for test data and This is based on the concept of measures of impurity of the data. These metrics can be entropy. Information gain is used to decide the class of each data item, which can be expresses as follows: [9], [10].

Gain $(R, A)=$ Entropy $(R)-\sum\left|R_{v}\right| /|R|$ Entropy $\left(R_{v}\right)$ '.

$R_{v}$ is the subset of $\mathrm{R}$ that has attribute value of $v$.

$\sum$ is over each attribute value of $A$.

$\left(R_{v}\right)$ the number of elements in the set $R_{v}$.

\section{OUR PRoposed ARCHITECTURAL REPRESENTATION}

The objective of our architecture is to achieve decision based on patient information.

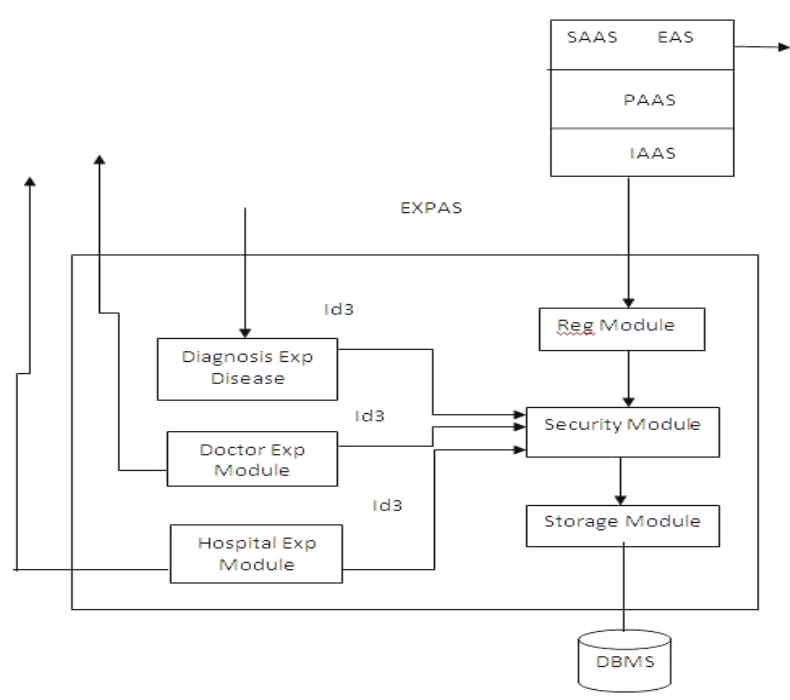

Fig. 1. Medical diagnosis expert system as service. 


\section{A. Our Architecture Consists of the Following Parts and Roles}

A model of cloud expert system as a service provides on demand in internet, eliminating the need for installing and maintenance of the expert system software on the clients computer. PAAS offer development tools. Ex: AZURE IAAs Virtual machines are provided Ex: Amazon. The patient who has full access to the cloud server.

A specific diagnostic expert system based on test reports.

A specific doctor expert system module need to diagnosis using ID3.

A specific Hospital expert system is to make decisions based on patient test reports.

Patient - centric control through cryptographic techniques can be applied and to maintain confidentiality and integrity of patient personal stores in the cloud need to be protected information.

Data in cloud through cloud service provides and if patient wants to access openly and other is to be provided security in terms of encryption techniques which secure patient information through decision for diagnosis can be made based on personal information of the patient and the data base, sends a request to the cloud service provide and receives the original data. If data is in encrypted form that can be decrypted in his key.

\section{B. Methodology of Expert System Service}

To provide patient information that will help the doctor makes critical decision concerning a patient health.

- If (Serum = 'Yellow') then do CD4 count test.

- Else 'No Disease Found'

- If (CD4 Count $\geq 500$ ) then 'patient in early stage'

- Else if (CD4 count <500 and CD4 Count >=200) and symptoms is appears then 'patient is in middle stage'.

- Else if (CD4<200 \& Symptoms appears then 'patient is critical stage')

- Else 'Patient is in Normal Stage'

\section{Decision Tree}

- Decision trees are simplistic.

- Decision trees and proven to be better classifier.

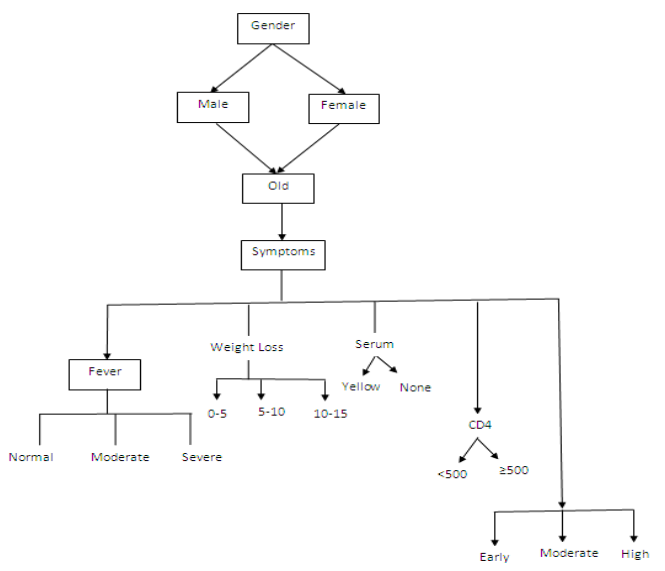

Fig. 2. Decision tree

To provide on demand - resource provision according to the service level established between a patient, a Doctor and Hospital. Access control can be made through registration by patients Doctors and Hospital During the process of decision making patient has to submit it his symptoms as input data. Diagnosis can be performed using Decision Tree. After disease prediction patient can choose specialized doctor. Selecting Hospital which chosen doctor treatment. And also can refer to medical insurance agents First and foremost patient has to submit his secret key to the cloud. Through which doctor can receives key data and suggests his views on that particular patient based on lab test reports. Diagnosis system is developed with the purpose of helping the doctor in diagnosis various diseases based on test reports Objective of this system into produce recovery data and information for decision making to the Doctor and with the results obtained to produce possible diagnosis.

\section{CONCLUSION}

In this paper, we developed a novel approach development of cloud medical diagnosis expert system as service, which provides secure patient information diagnosis based on lab test reports.

\section{REFERENCES}

[1] M. Deng, M. Petkovie, M. Nalin, and I. Baroni, "A home healthcare system in the cloud - addressing security and privacy challenges," in Proc. IEEE 4th International Conference on Cloud Computing, 2011, pp. 549-556.

[2] A. B. R. Kumar, C. P. Ramesh, E. Madhusudhan, and M. Padmavatamma, "Threshold Extended ID3 Algorithm," in Proc. ICDIP 2012 International Conference in Malaysia, 2012.

[3] B. Ried, T. Neubauer, and G. Goluch, "Secure Business Austria, a secure architecture for the pseudonymization of medical data," in Proc. Second International Conference on Availability Reliability and Security (ARES'07), 2007.

[4] L. Ibraimi, M. Asim, and I. Petkovie, "Secure Management of Personal Health Records by Applying Attribute-Based Encryption," Security and Privacy in Communication Networks, vol. 50, pp. 89-106, 2010.

[5] R. Ranchal, B. Bhargava, L. Ben Othmane, L. Lilien, A. Kim, M. Kang, and M. Linderman, "Protection of identity information in cloud computing without Trusted Third Party," in Proceedings of SRDS Symposium on Reliable Distributed Systems, pp. 368-372, 2010.

[6] R. Zhang and L. Liu, "Security models and requirements for healthcare application clouds," IEEE Cloud, pp. 268-275, 2010.

[7] J. R. Quinlan, Introduction of Decision Trees. Machine Learning, vol.1, pp. 81-106, 1986.

[8] A. N. M. Katongo, T. K. L. Dingalo, and D. Mpoeleng, "An expert system for HIV and AIDS information," in Proceedings of the World Congress on Engineering 2009, WCE 2009, London, U.K, vol. 1, July 1-3, 2009,

[9] F. Emekci, D. D. Sahin, D. Agarwal, and A. E. Abbadi, "Privacy preserving decision tree learning over multiple parties," Science Direct Data and Knowledge Engineering, pp. 348-361, 2007.

[10] M. Li, S. Yu, K. Ren, and W. Lon, "Securing Personal Health Records in Cloud Computing: Patient - Centric and Fine - Grained Data access Control in Multi - owner settings," Security and Privacy in Communication Networks, Social Informatics and Telecommunications Engineering, vol. 50, pp. 89-106, 2010.

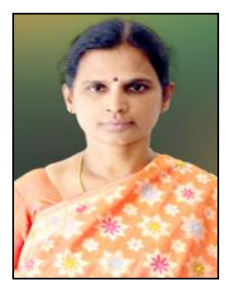

M. Padmavathamma is working as Head of Department of Computer Science, S.V.University, Tirupati, AP. India. She has vast experience of 26 years in teaching. She has guided 8 PhD's, 12 M.Phils and published 35 articles in International/National Journals. She has attended and chaired many International conferences conducted by various International organizations at various places around the world Currently she is director of projects funded by UGC, DST India. Her Areas of interest are Network Security, Cloud computing and Data Mining. 


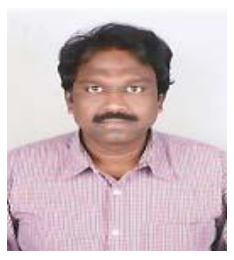

A. B. Rajesh Kumar is a research scholar from S.V.University Department of Computer Science, Tirupati, AP, India and presented papers and attended international conferences in India and around Asia. His areas of interest are Network security and Data mining.

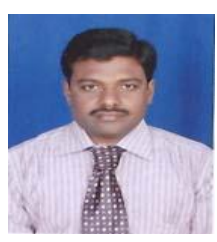

G. V. Ramesh Babu is working as Asst.Professor, Department of computer science, S.V.University, Tirupati. AP, India . He has a teaching experience of 13 years and presented papers and attended international conferences in India and around Asia. His areas of interest are Network security, Ecommerce and Data mining.

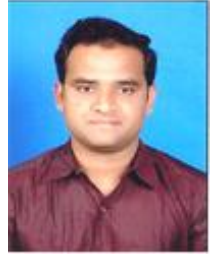

C. Phani Ramesh is a research scholar from S.V University Department of Computer Science, Tirupati, AP, India and presented papers and attended international conferences in India and around Asia. His areas of interest are Network Security, Ecommerce and Data mining.

P. Madhura is a research Scholar from S.V. University Department of Computer Science, Tirupati, AP, India. Her areas of interest are Network security and Data mining. 